\title{
More words in the neighborhood: Interference in lexical decision due to deletion neighbors
}

\author{
COLIN J. DAVIS \\ Macquarie Centre for Cognitive Science, Sydney, New South Wales, Australia \\ and \\ MARCUS TAFT \\ University of New South Wales, Sydney, New South Wales, Australia
}

\begin{abstract}
This article reports two lexical decision experiments that provide evidence for the automatic activation of deletion neighbors - that is, words that overlap with the presented word save for the deletion of one letter. Experiment 1 showed slower and less accurate no decisions for nonwords with deletion neighbors (e.g., come in scome), relative to control nonwords. Experiment 2 showed slower and less accurate yes decisions for words with higher frequency deletion neighbors, relative to control words. An important methodological implication of these results is that stimuli should be equated using a different definition of orthographic neighborhood from that which is currently the norm. The results also have significant theoretical implications for input coding schemes and the mechanisms underlying recognition of familiar words.
\end{abstract}

The effects of orthographic similarity on visual word identification have attracted considerable theoretical and empirical attention (for a review, see Andrews, 1997). The research suggests that processing of a written word results in the automatic activation of orthographically similar words (the word's orthographic neighbors) and that this can affect the speed of lexical access. Establishing which words make up the similarity neighborhood of a letter string can offer valuable insights into the structure of lexical representations and the nature of input coding. Theoretical input coding schemes make predictions about the similarity of pairs of letter strings and, hence, which words are likely to be activated following presentation of a target letter string. For example, some coding schemes predict that the words train and rain are coded in such a way that there is little or no overlap in their respective input codes; such schemes can be falsified if evidence is found that the representation of rain is automatically activated following presentation of train. Another key question concerns whether the activation of orthographic neighbors facilitates or inhibits word recognition; the answer to this question is critical for understanding the mechanisms underlying word identification. The present article reports two experiments that bear upon these issues.

\footnotetext{
We gratefully acknowledge Derek Hanley and Annukka Lindell for their help with data collection. Helpful feedback on an earlier draft of the manuscript was provided by Dave Balota, Jeff Bowers, Max Coltheart, and Michael Cortese. Correspondence concerning this article should be addressed to C. J. Davis, Department of Experimental Psychology, University of Bristol, 8 Woodland Rd., Bristol BS8 1TN, England (e-mail: colin.davis@bristol.ac.uk).
}

The first lexical decision experiment in which orthographic neighborhood effects were investigated was reported by Coltheart, Davelaar, Jonasson, and Besner (1977). They examined the effect of a metric that they called $N$, which is a count of the number of substitution neighbors (SNs) of a letter string - the number of words sharing the same letter in all but one position. They found that $N$ had no effect on the latency of yes responses, but that no responses to large- $N$ nonwords were slower than those to small- $N$ nonwords. A wealth of subsequent research has examined the effect on stimulus recognition of both the size of the similarity neighborhood and the frequency of the words contained in the neighborhood. Virtually all of this research has adopted the definition of orthographic neighbors employed by Coltheart et al (1977).

However, the $N$ metric is probably only an approximate measure of the size of a word's (or a nonword's) similarity neighborhood. The first indication of this was provided by evidence of transposed letter similarity effects (e.g., Andrews, 1996; Chambers, 1979). A transposition neighbor (TN) of a letter string is a word that is identical save for the transposition of two adjacent letters. The confusability of TN pairs such as clam and calm suggests that the definition of a letter string's neighborhood should be broadened to include not just words that can be formed by letter substitution, but also words formed by letter transpositions.

In this article, we present evidence for a further broadening of the similarity neighborhood to include words formed by letter deletions. We define a deletion neighbor (DN) of a word to be a letter string that differs from that word by the deletion of a single letter; for example, the DNs of plane are plan, pane, and lane. The conventional measure of orthographic similarity counts includes SNs such as place and 
plant in the word plane's orthographic neighborhood but excludes DNs such as plan and lane, despite the fact that these words are fully contained within the word plane.

Given the extensive literature on the similarity of SNs, surprisingly few studies have taken into consideration the orthographic similarity of words that differ in length (although see Treiman, Mullennix, Bijeljac-Babic, \& RichmondWelty, 1995; van Heuven \& Dijkstra, 2005; Ziegler \& Perry, 1998), and even fewer have taken into consideration the similarity of DNs (Bowers, Davis, \& Hanley, 2005a; De Moor \& Brysbaert, 2000; Drews \& Zwitserlood, 1995). De Moor and Brysbaert found an inhibitory effect of masked word primes that were DNs of the targets (e.g., rover-Dutch for robber - took longer to classify as a word when it was preceded by the DN over than when it was preceded by the unrelated word prime zich). A similar finding was reported by Drews and Zwitserlood, using German stimuli. These findings are consistent with the idea that identification of a target word may be delayed by competition with its DNs; priming the target with a DN enhances this competition. However, the fact that the DNs are presented in this paradigm (albeit briefly) makes it difficult to conclude that DNs are automatically activated in normal reading.

Drews and Zwitserlood (1995) also reported another priming experiment in which they observed no priming from nonword primes that were supersets of the target word (e.g., pilst-pil). If DNs are automatically activated, it might be expected that these primes would facilitate target processing, in the same way that other masked priming experiments have shown that nonword primes that are SNs or TNs of a target word facilitate lexical decisions to that target (e.g., Forster, Davis, Schoknecht, \& Carter, 1987). The absence of priming in this experiment may reflect the fact that the targets were not from low-density neighborhoods, which appears to be critical for obtaining facilitatory masked priming (Forster et al., 1987); it should also be noted that the primes were often several letters longer than the target and, hence, that the stimuli did not provide an ideal test of the similarity of DNs, as defined here. In any case, it must be concluded that priming experiments have yet to provide strong evidence for the automatic activation of DNs.

More convincing evidence has recently been reported by Bowers et al. (2005a), who observed interference effects from DNs in semantic categorization. For example, participants took longer to respond no when asked to decide whether the word apex (which has the DN ape) refers to a type of animal than when asked to decide whether apex refers to a type of vehicle. This interference effect suggests that DNs were processed to the level of meaning and impaired performance when they required a different response than the presented word.

As Bowers et al. noted, their results do not bear on the question of whether DNs compete with their superset words at the lexical level. The experiments reported in the present article used the lexical decision task (LDT) and may thus offer a more direct test of the dual claims that DNs are automatically activated during visual word recognition and that they compete with the superset words within which they are contained.

\section{EXPERIMENT 1}

Previous LDT studies have shown that a single SN or TN is sufficient to produce an inhibitory effect on no responses to nonwords (e.g., Chambers, 1979; Forster \& Shen, 1996). In the present experiment, we examined whether there is a similar inhibitory effect of orthographic similarity for nonwords that possess DNs, relative to matched control nonwords. We hypothesized that the lexical representations of DNs would be automatically activated during the processing of nonword stimuli (e.g., the nonword smade would lead to the automatic activation of its DN made) and that this lexical activation would interfere with no responses, resulting in slower responses than to control nonwords such as smoad. ${ }^{1}$

\section{Method}

Participants. Thirty-one undergraduate students from the University of Bristol participated. All were native English language speakers and had normal or corrected-to-normal vision.

Stimuli and Design. There were two conditions, each consisting of 26 five-letter pronounceable nonwords (the full list can be found in the Appendix). Nonwords in the DN condition consisted of an initial letter that was followed by a four-letter word (e.g., smade). The DN word (in this case, made) had a CELEX written frequency of at least 10 per million; the median frequency was 170 per million. The combination of the nonword's initial letter and the initial letter of the four-letter word always resulted in a legal onset. To ensure perfect matching of onset and body units across the two nonword conditions, quadruples of items were selected consisting of two onset units and two body units. For example, the nonwords smade and droad in the DN condition were matched with the nonwords smoad and drade in the control condition; nonwords in the control condition had no DNs. The DN and control nonwords in each quadruple were matched with respect to $N(M=1.60$ and 1.53 for the $\mathrm{DN}$ and control conditions, respectively). The two conditions were also matched on mean $\log$ bigram frequency $(M=2.55$ and 2.57 , respectively) and mean $\log$ trigram frequency $(M=1.46$ and 1.35 , respectively); values for these variables were calculated using the $\mathrm{N}$-Watch computer program (Davis, 2005a). There were 80 filler stimuli: 52 five-letter words, 14 four-letter words, and 14 four-letter nonwords. The mean CELEX written frequency of these words was 39 per million (range $=2-472$ ), and the mean $N$ was 5 (range $=$ $0-17)$. None of the four-letter words corresponded to the DNs that were embedded in the critical nonword condition.

Procedure. The participants were tested in a quiet room either individually or in groups of 2 or 3 . They were told that words and nonwords would be displayed on the monitor in front of them and that they should press one of two buttons to indicate whether each stimulus was a word or a nonword, responding as rapidly as possible while maintaining a reasonable level of accuracy. Word responses were made with the dominant hand. The participants were initially presented with 20 sample trials consisting of 10 words and 10 nonwords. The experiment started with 3 filler trials, followed by the 132 experimental trials, the order of which was randomized for each participant. The stimuli were presented in lowercase, using the DMDX software for stimulus display (K. I. Forster \& J. C. Forster, 2003), and remained visible until the participant responded.

\section{Results and Discussion}

Latencies greater than $1,500 \mathrm{msec}(0.4 \%$ of the data) or less than $300 \mathrm{msec}(0 \%)$ were excluded from the analysis, 
as were the data from 1 participant who had a very high error rate $(39 \%){ }^{2}$ For word stimuli, the mean response time (RT) was $604 \mathrm{msec}$, and the mean error rate was .06. Two-tailed repeated measures $t$ tests on the difference between the two nonword conditions showed that nonwords with DNs were classified more slowly $(669 \mathrm{msec}, S E=$ 20.30) than control nonwords [649 $\mathrm{msec}, S E=18.56$; $t_{1}(29)=2.96, M S_{\mathrm{e}}=457.02, p<.01 ; t_{2}(25)=2.47$, $\left.M S_{\mathrm{e}}=776.69, p<.05\right]$. The error rate was also higher for nonwords with DNs $(.07, S E=.01)$ than for control nonwords [.04, $S E=.01 ; t_{1}(29)=2.69, M S_{\mathrm{e}}=.001, p<$ $\left..05 ; t_{2}(25)=1.61, M S_{\mathrm{e}}=.003, p<.12\right]$.

The inhibitory effect of DNs in this experiment parallels the effect of SNs and TNs on nonwords in other LDT experiments (e.g., Chambers, 1979; Forster \& Shen, 1996), and it seems reasonable to conclude that these phenomena reflect the same underlying effect, whereby the similarity of a nonword to existing words gives rise to lexical activity that interferes with the correct no classification.

It is possible, however, that the DN interference effect is specific to nonword stimuli-that is, that DNs are activated only when the lexical processor fails to find a lexical representation matching the input stimulus. To examine this possibility, we sought in Experiment 2 to find evidence of automatic DN activation for word stimuli.

\section{EXPERIMENT 2}

Experiments with French, Dutch, and Spanish materials have shown that the presence of a higher frequency neighbor inhibits the latency of yes responses to word stimuli (e.g., Grainger \& Jacobs, 1996; Grainger, O'Regan, Jacobs, \& Segui, 1989, 1992). This effect has been replicated with English stimuli in some studies (e.g., Huntsman \& Lima, 1996; Perea \& Pollatsek, 1998), but not in others (e.g., Forster \& Shen, 1996; Sears, Hino, \& Lupker, 1995). These empirical inconsistencies may be related, in part, to the use of an overly conservative definition of a word's orthographic neighborhood; this possibility will be discussed below. They probably also reflect the difficulty of matching low-frequency stimuli satisfactorily on all relevant variables (e.g., Forster \& Shen, 1996). For this reason, methodologies that enable a target word to be compared with itself are of particular value; a variety of such techniques have shown inhibitory effects of higher frequency SNs in English. For example, briefly delaying presentation of one of the letters of a target word has a significantly greater inhibitory effect on lexical decision latency when this results in a letter string that is potentially consistent with a higher frequency neighbor, as compared with one that is unambiguously consistent with the target (e.g., delaying the $d$ in drew-enabling activation of the higher frequency neighbor grew-results in much longer latencies than does delaying the letter $w$; Hinton, Liversedge, \& Underwood, 1998; Pugh, Rexer, Peter, \& Katz, 1994). More recently, Bowers, Davis, and Hanley (2005b) have used a training methodology in which new words are introduced into readers' lexicons, so that it is possible to examine performance on the same word when it is a lexical hermit and when it has an orthographic neighbor; results showed that the presence of a new neighbor exerted an inhibitory effect on the speed of semantic categorizations and lexical decisions.

On the basis of these considerations, we predicted that the presence of a higher frequency DN would exert an inhibitory effect on lexical decisions to low-frequency words. To test this, we selected low-frequency words with higher frequency DNs (e.g., comet, stable) and matched these as closely as possible to control words with no DNs. We also varied the position of the overlap between a word and its DN. Bowers et al. (2005a) observed no difference in the magnitude of the interference effect for wordinitial and word-final embedded subsets (e.g., the embedded word arm interfered with decisions to both army and farm). We attempted to confirm that interference from DNs can be obtained across both of these position conditions and also included a third condition, in which the DN overlapped with the outer portion of a word (e.g., trace in trance). This type of overlap involves a disruption of letter contiguity but preserves the exterior letters of the DN. In the light of evidence supporting the greater weight of exterior letters in visual word identification (e.g., Humphreys, Evett, \& Quinlan, 1990; Jordan, 1990), we expected that interference would also be observed in this condition.

\section{Method}

Participants. Twenty-eight undergraduate students from the University of New South Wales participated. All were native English language speakers and had normal or corrected-to-normal vision.

Stimuli and Design. The stimuli consisted of 240 items: 120 low-frequency words (mean CELEX frequency $=8$ per million) and 120 nonwords. Half of the word stimuli possessed DNs that were of higher frequency than the stimulus word (mean CELEX frequency $=318$ per million). The position of the DN word was varied factorially across three levels: (1) initial overlap (e.g., tablet), (2) final overlap (e.g., turban), and (3) outer overlap (e.g., trance). For each of these conditions, $60 \%$ of the words had five letters, and $40 \%$ had six.

Each of the critical words was paired with a control word that did not possess a DN. A number of the control words contained smaller embedded words (e.g., are in snare, bat in batch). This is inevitable given the degree of lexical embedding in English and the constraints on frequency matching; the important point to note is that the influence of these subset words should act against the hypothesis being tested here. Experimental and control words were matched with respect to length, number of syllables, $N$, bigram frequency, and word frequency (see Table 1). To ensure close frequency matches between experimental and control items, three different written word frequency norms were employed: the CELEX English Corpus Types database (Baayen, Piepenbrock, \& van Rijn, 1995), Kučera and Francis (1967), and the Sydney Morning Herald word corpus (Dennis, 1995). The conditions were also matched with respect to $N$ and bigram frequency, using values calculated by the $\mathrm{N}$-Watch computer program (Davis, 2005a). None of the stimuli possessed TNs. The nonwords were filler stimuli that resembled the word stimuli with respect to their orthographic characteristics (e.g., 60\% contained five letters, the remainder contained six letters, and the distribution of $N$ values was similar to that for the word stimuli).

Procedure. The procedure in this experiment was identical to that in Experiment 1, except that the participants were always tested individually. 
Table 1

Characteristics of the Word Stimuli Employed in Experiment 2

\begin{tabular}{lllccccc}
\hline DN Condition & \multicolumn{1}{c}{ Examples } & Neletion & & & & & \\
\hline Initial overlap & overt, tablet & over, table & 7.6 & 9.5 & 8.6 & 2.7 & 2.7 \\
Matched control & exert, tumble & & 7.9 & 8.4 & 8.4 & 2.9 & 2.6 \\
Final overlap & blast, turban & last, urban & 9.1 & 7.7 & 6.1 & 2.5 & 2.8 \\
Matched control & blade, tremor & & 9.1 & 8.1 & 6.5 & 2.5 & 2.8 \\
Outer overlap & drown, trance & down, trace & 7.1 & 7.1 & 5.8 & 1.3 & 2.7 \\
Matched control & clown, thirst & & 7.5 & 7.1 & 6.3 & 1.5 & 2.7 \\
\hline
\end{tabular}

Note-DN, deletion neighbor; CELEX, CELEX written word frequency corpus; KF, Kučera-Francis (1967) written word frequency corpus; SMH, Sydney Morning Herald written word frequency corpus; $N$, neighborhood size; MLBF, mean log bigram frequency. All frequencies refer to mean number of occurrences per million words.

\section{Results and Discussion}

Mean correct RTs and error rates (averaged over participants) are shown in Table 2. Latencies greater than $1,500 \mathrm{msec}(1.5 \%$ of the data) or less than $300 \mathrm{msec}(0 \%)$ were excluded from the analysis, as were the data from 1 participant whose error rate exceeded $30 \% .{ }^{3}$ Differences between DN and control words were tested over both participants and items, using repeated measures analyses in both cases. Words with higher frequency DNs were classified $27 \mathrm{msec}$ more slowly than matched control words $\left[F_{1}(1,26)=31.86, M S_{\mathrm{e}}=911.11, p<.01 ; F_{2}(1,57)=\right.$ $\left.7.57, M S_{\mathrm{e}}=3,334.76, p<.01\right]$. This DN interference effect interacted with position of overlap in the analysis over participants $\left[F_{1}(1,25)=3.69, M S_{\mathrm{e}}=1,069.83, p<.05\right]$, but not in the analysis over items $(F<1)$. There were no significant differences in the analyses of the error data. For nonword stimuli, the mean RT was $719 \mathrm{msec}$, and the mean error rate was .10.

Although the stimuli were closely matched on frequency and orthographic variables, we did not attempt to match with respect to semantic variables, such as word imageability, when selecting stimuli, and this raises the possibility that the observed effect could reflect a confound with imageability. ${ }^{4}$ To assess this possibility, we collected ratings of imageability (on a scale from 1 to 7 ) for each of the words in the experiment from a sample of 10 raters drawn from the same student population as the participants in Experiment 2. Although the difference in mean imageability between conditions was not large (4.4 vs. 3.7$)$, it was statistically significant $(p=.03)$. We therefore conducted a new analysis over the matched item pairs, in which we included the difference in mean imageability ratings as a covariate. This resulted in the same pattern of effects for the RT data, with a significant DN interference effect $\left[F_{2}(1,56)=4.05, M S_{\mathrm{e}}=3,026.91, p<\right.$ $.05]$ and no interaction with position of overlap $(F<1)$; there were no differences in the error rate data, as in the original analysis. It can be concluded that there is a genuine DN interference effect above and beyond any effect that is due to imageability.

In order to test whether differences in the pronunciation of the target and its deletion neighbor affected the results, we conducted a post hoc analysis in which we divided the stimulus pairs up according to whether the pronunciation of the DN was the same as when it was presented in the target word (e.g., star and stark were classified as same, whereas close and closet were classified as different). ${ }^{5}$ Mean DN interference was $30 \mathrm{msec}$ for same pronunciation items $(n=36)$ and $25 \mathrm{msec}$ for different pronunciation items $(n=24)$; the interaction between the pronunciation factor and the DN interference effect did not approach significance $\left[F_{2}(1,54)=1.29, p>.25\right]$. It therefore appears that this pronunciation factor does not influence the interference effect of DNs. This agrees with the conclusion drawn by Bowers et al. (2005a).

A final post hoc analysis examined whether DN interference was moderated by the number of substitution neighbors of the target. According to competitive activation models, the inhibitory effects of a given competitor should be masked when there are multiple competitors. We divided the items into two roughly equally sized groups, one consisting of 29 targets with either no neighbors or one (lower frequency) neighbor, another consisting of 31 targets that had between two and nine neighbors. For the low- $N$ group the DN interference was particularly strong (44 msec, $p=.001$ ), but for the group with multiple neighbors the DN interference effect was much weaker $(13 \mathrm{msec})$ and not statistically significant $(p>.4){ }^{6}$

Overall, the results were consistent with the predictions: Higher frequency DNs interfered with lexical decision, and there was evidence of this interference effect for word-initial, word-final, and word-outer overlap condi-

Table 2

Mean Response Times (RTs, in Milliseconds) and Error Rates (ERs) for Experiment 2

\begin{tabular}{|c|c|c|c|c|c|}
\hline \multirow{2}{*}{$\begin{array}{l}\text { Overlap } \\
\text { Condition }\end{array}$} & \multirow{2}{*}{$\begin{array}{c}\text { DN } \\
\text { Condition }\end{array}$} & \multicolumn{2}{|c|}{ RT } & \multicolumn{2}{|c|}{ ER } \\
\hline & & $M$ & $S E$ & $M$ & $S E$ \\
\hline \multirow[t]{2}{*}{ Initial } & $\mathrm{DN}$ & 667 & 19.0 & .05 & .01 \\
\hline & $\begin{array}{l}\text { Control } \\
\text { Difference }\end{array}$ & $\begin{array}{r}639 \\
28\end{array}$ & 17.4 & $\begin{array}{c}.05 \\
0\end{array}$ & .01 \\
\hline \multirow[t]{2}{*}{ Final } & $\mathrm{DN}$ & 617 & 13.9 & .04 & .01 \\
\hline & $\begin{array}{l}\text { Control } \\
\text { Difference }\end{array}$ & $\begin{array}{r}608 \\
9\end{array}$ & 16.8 & $\begin{array}{c}.04 \\
0\end{array}$ & .01 \\
\hline \multirow[t]{2}{*}{ Outer } & $\mathrm{DN}$ & 639 & 16.6 & .05 & .01 \\
\hline & $\begin{array}{l}\text { Control } \\
\text { Difference }\end{array}$ & $\begin{array}{r}595 \\
44\end{array}$ & 15.1 & $\begin{array}{c}.05 \\
0\end{array}$ & .01 \\
\hline
\end{tabular}

Note-DN, deletion neighbor. 
tions. The data showed a strong trend for greater interference for the latter condition, although the interaction between DN interference and position of overlap was not robust: It was observed in the analysis over participants when an absolute criterion was used to exclude outliers, but not when a relative criterion was used (see note 3 ), nor was it apparent in the analyses over items.

\section{GENERAL DISCUSSION}

The present experiments provide evidence that the presentation of a target letter string leads to the automatic activation of DNs and, therefore, support the notion that the definition of a letter string's similarity neighborhood should be extended to include DNs. Although it may seem unsurprising that words such as plane and plan are perceptually similar, this finding has important methodological and theoretical implications. Previous research indicates that the similarity neighborhood of a letter string affects the speed with which that letter string can be processed in tasks such as lexical decision, speeded naming, and perceptual identification (e.g., Andrews, 1997). For this reason, it is standard practice to equate stimuli on $N$ when investigating the effects of other variables, on the assumption that $N$ is a measure of the density of a letter string's similarity neighborhood. But if the standard $N$ metric is only a rough measure of neighborhood density, the possibility exists that efforts to control stimuli with respect to similarity will be unsuccessful.

From a theoretical perspective, it is also critical to establish which words make up the similarity neighborhood of a letter string, because this bears upon the nature of the input coding scheme employed by the word recognition system. The most common form of input coding scheme in current computational models is some form of positionspecific coding, in which it is assumed that there are letter units that correspond to particular serial positions. These schemes are well suited to explaining the similarity of SNs but face problems explaining the similarity of TNs (e.g., Andrews, 1996; Davis, 1999), as well as DNs in which the common letters are misaligned. For example, the results of Experiments 1 and 2 suggest that the presentation of either the nonword scome or the word comet results in the automatic activation of the high-frequency DN come. The similarity of scome and come cannot be explained by a simple position-specific coding scheme such as that used in the DRC model (Coltheart, Rastle, Perry, Langdon, \& Ziegler, 2001), because scome and come are not coded by any common letter units. Switching to a rightaligned form of slot coding would overcome this problem but would then fail to explain the similarity of DNs that involve word-initial overlap (e.g., comet and come). Finding a coding scheme that can accommodate these effects is an important problem that needs to be solved by computational models of visual word recognition.

One candidate scheme that does just this has been described by Davis (1999, 2005b, in press). A key aspect of this spatial coding scheme is its assumption of positionindependent letter units; the relative activities across these units code the relative order of the letters in a string. This implies that words such as plane, plan, lane, and pane are coded by similar patterns of activity across the same set of letter units and, hence, that they will occupy a common similarity neighborhood. Davis (2005b) has suggested possible extensions to this scheme to explain data suggesting that exterior letters play a more important role in visual word identification than do interior letters (e.g., Humphreys et al., 1990; Jordan, 1990). One possibility is that exterior letters tend to be identified earlier than interior letters (because they suffer from less lateral masking), so that word identification is characterized by outside-in processing (e.g., Grainger et al., 1992; Jordan, 1990). This leads to the prediction that the input stimulus is almost twice as similar to an outer-overlap DN than to an initial or final DN for the period during which only the exterior letters have been identified. ${ }^{7}$ This may account for the numerical trend we observed in Experiment 2 in the direction of greater interference for DNs involving wordouter overlap.

A final critical issue raised by the present data concerns the question of whether neighbors facilitate or inhibit the processing of a letter string. In the case of nonwords, the answer to this question is clear: Whether the neighbor is formed by letter substitution, transposition, or deletion (as in Experiment 1), the effect of lexical neighbor activation is to interfere with no responses in LDT, resulting in slower and less accurate responses (Andrews, 1996; Chambers, 1979; Forster \& Shen, 1996). This is consistent with the idea that the mechanism for making no decisions in LDT monitors on-line lexical activity (e.g., Coltheart et al., 1977; Davis, 1999; Grainger \& Jacobs, 1996). In the case of words, the answer is less clear; as was noted earlier, experiments in which English stimuli have been used have produced mixed results. One factor contributing to the absence of an inhibitory effect of higher frequency SNs in some experiments may be the difficulty of adequately matching stimulus conditions across all the relevant variables. In the present experiment, a very strict method of matching conditions with respect to word frequency was used, and post hoc analyses revealed that the inhibitory effect of higher frequency DNs was still obtained when differences in the imageability of the conditions were covaried out. Another factor contributing to the mixed empirical results may be related to the methodological issue raised by the present data-that is, the use of an overly conservative definition of a word's orthographic neighborhood. For example, a word such as acre has no higher frequency SNs, but it has both a higher frequency TN (care) and a higher frequency DN (are) and is, therefore, an inappropriate control item to use in an experiment examining whether words with higher frequency neighbors are identified more slowly. By the same logic, DN interference effects should be harder to detect for words that have multiple SNs. This is exactly what we found in the post hoc analysis in Experiment 2, which revealed that the DN interference effect was very robust for words with no SNs (or one lower frequency SN), but not for words with multiple SNs (a result that parallels the 
results of training experiments reported by Davis, Bowers, \& Hanley, 2005). This pattern supports the prediction of competitive network models in which identification is achieved through competition among lexical representations in the similarity neighborhood of the input stimulus. The present work shows that this neighborhood needs to be extended to include DNs.

\section{REFERENCES}

ANDREWs, S. (1996). Lexical retrieval and selection processes: Effects of transposed-letter confusability. Journal of Memory \& Language, 35, 775-800.

ANDREws, S. (1997). The effect of orthographic similarity on lexical retrieval: Resolving neighborhood conflicts. Psychonomic Bulletin \& Review, 4, 439-461.

BaAyen, R. H., Piepenbrock, R., \& VAn RiJn, H. (1995). The CELEX Lexical Database: Release 2 [CD-ROM]. Philadelphia: University of Pennsylvania, Linguistic Data Consortium.

Bowers, J. S., Davis, C. J., \& Hanley, D. A. (2005a). Automatic semantic activation of embedded words: Is there a "hat" in "that"? Journal of Memory \& Language, 52, 131-143.

Bowers, J. S., Davis, C. J., \& Hanley, D. A. (2005b). Interfering neighbours: The impact of novel word learning on the identification of visually similar words. Cognition, 97, 45-54.

Chambers, S. M. (1979). Letter and order information in lexical access. Journal of Verbal Learning \& Verbal Behavior, 18, 225-241.

Coltheart, M., Davelaar, E., Jonasson, J. T., \& Besner, D. (1977). Access to the internal lexicon. In S. Dornic (Ed.), Attention and performance VI (pp. 535-555). New York: Academic Press.

Coltheart, M., Rastle, K., Perry, C., Langdon, R., \& Ziegler, J. (2001). DRC: A dual route cascaded model of visual word recognition and reading aloud. Psychological Review, 108, 204-256.

DAVIS, C. J. (1999). The self-organising lexical acquisition and recognition (SOLAR) model of visual word recognition. Unpublished doctoral dissertation. Available at www.maccs.mq.edu.au/ colin.

DAVIS, C. J. (2005a). N-Watch: A program for deriving neighborhood size and other psycholinguistic statistics. Behavior Research Methods, 37, 65-70.

DAVIS, C. J. (2005b). The SOLAR (self-organising lexical acquisition and recognition) model of visual word identification, Pt. I: Orthographic input coding and lexical matching. Manuscript submitted for publication.

DAvIS, C. J. (in press). Orthographic input coding: A review of behavioural data and current models. In S. Andrews (Ed.), From inkmarks to ideas: Current issues in lexical processing. Hove, U.K.: Taylor \& Francis, Psychology Press.

Davis, C. J., Bowers, J. S., \& HANLey, D. A. (2005, January). Interfering neighbours: The impact of novel word learning on the identification of orthographically similar words. Poster presented at the meeting of the Experimental Psychology Society, London.

De Moor, W., \& Brysbaert, M. (2000). Neighborhood-frequency effects when primes and targets are of different lengths. Psychological Research, 63, 159-162.

Dennis, S. (1995). The Sydney Morning Herald Word Database. Noetica: Open Forum, 1(4), http://psy.uq.edu.au/CogPsych/Noetica/.

Drews, E., \& ZwitsERLOOD, P. (1995). Morphological and orthographic similarity in visual word recognition. Journal of Experimental Psychology: Human Perception \& Performance, 21, 1098-1116.

Forster, K. I., Davis, C., Schoknecht, C., \& Carter, R. (1987). Masked priming with graphemically related forms: Repetition or partial activation? Quarterly Journal of Experimental Psychology, 39A, 211-251.

Forster, K. I., \& Forster, J. C. (2003). DMDX: A Windows display program with millisecond accuracy. Behavior Research, Methods, Instruments, \& Computers, 35, 116-124.

Forster, K. I., \& SHEN, D. (1996). No enemies in the neighborhood: Absence of inhibitory neighborhood effects in lexical decision and semantic categorization. Journal of Experimental Psychology: Learning, Memory, \& Cognition, 22, 696-713.

Grainger, J., \& JacoBs, A. M. (1996). Orthographic processing in visual word recognition: A multiple read-out model. Psychological Review, 103, 518-565.

Grainger, J., O’Regan, J. K., Jacobs, A. M., \& Segui, J. (1989). On the role of competing word units in visual word recognition: The neighborhood frequency effect. Perception \& Psychophysics, 45, 189-195.

Grainger, J., O’Regan, J. K., Jacobs, A. M., \& Segui, J. (1992). Neighborhood frequency effects and letter visibility in visual word recognition. Perception \& Psychophysics, 51, 49-56.

Hinton, J., Liversedge, S. P., \& Underwood, G. (1998). Neighborhood effects using a partial priming methodology: Guessing or activation? Journal of Experimental Psychology: Learning, Memory, \& Cognition, 24, 1294-1305.

Humphreys, G. W., Evett, L. J., \& Quinlan, P. T. (1990). Orthographic processing in visual word identification. Cognitive Psychology, 22, 517-560.

Huntsman, L. A., \& Lima, S. D. (1996). Orthographic neighborhood structure and lexical access. Journal of Psycholinguistic Research, 25, 417-429.

Jordan, T. R. (1990). Presenting words without interior letters: Superiority over single letters and influence of postmask boundaries. Journal of Experimental Psychology: Human Perception \& Performance, 16, 893-909.

KuČERA, H., \& Francis, W. N. (1967). Computational analysis of presentday American English. Providence, RI: Brown University Press.

Perea, M., \& PollatseK, A. (1998). The effects of neighborhood frequency in reading and lexical decision. Journal of Experimental Psychology: Human Perception \& Performance, 24, 767-779.

Pugh, K. R., Rexer, K., Peter, M., \& Katz, L. (1994). Neighborhood effects in visual word recognition: Effects of letter delay and nonword context difficulty. Journal of Experimental Psychology: Human Perception \& Performance, 20, 639-648.

Sears, C. R., Hino, Y., \& Lupker, S. J. (1995). Neighborhood size and neighborhood frequency effects in word recognition. Journal of Experimental Psychology: Human Perception \& Performance, 21, 876-900.

Treiman, R., Mullennix, J., Bijeljac-Babic, R., \& RichmondWelty, E. D. (1995). The special role of rimes in the description, use, and acquisition of English orthography. Journal of Experimental Psychology: General, 124, 107-136.

van Heuven, W. J. B., \& Dijkstra, A. (2005). Extended neighborhood effects in visual word recognition. Poster presented at the XIV meeting of the European Society for Cognitive Psychology, Leiden.

Ziegler. J. C., \& Perry, C. (1998). No more problems in Coltheart's neighborhood: Resolving neighborhood conflicts in the lexical decision task. Cognition, 68, 53-62.

\section{NOTES}

1. We are grateful to Mike Cortese for suggesting the idea that led to this experiment.

2. A separate set of analyses in which RTs greater than 1,500 msec were replaced with RTs of $1,500 \mathrm{msec}$ resulted in the same pattern of significant results; furthermore, the same pattern of results was also observed when the participant with a very high error rate was retained in the analysis.

3. A separate set of analyses in which RTs greater than $1,500 \mathrm{msec}$ were replaced with RTs of $1,500 \mathrm{msec}$ resulted in the same pattern of significant results. Likewise, the pattern of significant results was unchanged when the participant with a high error rate was included in the analyses. Finally, we also conducted participant and item analyses in which RTs were excluded only if they were more than $2.5 \mathrm{SDs}$ from the mean for that participant/item. This resulted in a very similar pattern of effects; the only difference was that the interaction of DN interference and position of overlap did not quite attain significance in the analysis over participants $\left[F_{1}(2,25)=2.67, p=.08\right]$.

4. We thank Mike Cortese for pointing this out.

5. We thank Dave Balota for suggesting this analysis.

6 . The same pattern of significant results was obtained when effects of imageability were partialled out.

7. The details of these similarity calculations can be found in Davis (2005b). The exact ratio reflecting the similarity of outer overlap DNs relative to initial and final DNs depends on the setting of a parameter that reflects the degree of position uncertainty in the input code; the value used in simulations results in a ratio of 1.84 . 


\section{Nonword Stimuli in Experiment 1}

smade, smoad, droad, drade; dwait, dwike, clike, clait; dwish, dwift, clift, clish; croof, crive, glive, gloof; plamb, plich, frich, framb; frude, frike, plike, plude; smade, smard, thard, thade; scome, scoad, pload, plome; swith, swoom, troom, trith; skeep, skush, grush, greep; spath, spude, grude, grath; preal, preaf, gleaf, gleal; drisk, drist, clist, clisk

Note-Stimuli are arranged in quadruples of the form \{DN 1, Control 1, DN 2, Control 2\}, where DN 1 and Control 1 share the same onset, DN 1 and Control 2 share the same body, DN 2 and Control 2 share the same onset, and DN 2 and Control 1 share the same body. DN refers to nonwords with deletion neighbors.

Word Stimuli in Experiment 2

\begin{tabular}{llllll}
\hline Initial DN & Control & Final DN & Control & Outer DN & Control \\
\hline drawl & snare & swarm & sneak & truce & sniff \\
plank & batch & pinch & blunt & merge & blaze \\
overt & exert & brisk & cheer & drown & clown \\
stark & hatch & blast & blade & niece & brute \\
easel & nasal & groom & scalp & ounce & stump \\
comet & pivot & drain & stove & rinse & brood \\
cello & chime & grope & prune & bowel & urine \\
facet & fauna & crush & bliss & arena & choir \\
camel & dummy & bless & shaft & width & arrow \\
realm & gloom & sword & cough & crook & chalk \\
suite & verse & yeast & frost & seize & wreck \\
beard & vague & trace & shade & spoil & torch \\
median & umpire & twitch & trench & derive & locate \\
saucer & beetle & sentry & ripple & hostel & hockey \\
treaty & copper & soften & shiver & clause & freeze \\
superb & summit & praise & stroke & strand & sleeve \\
tablet & tumble & turban & tremor & statue & custom \\
ration & ribbon & trifle & pepper & trance & thirst \\
closet & solemn & plight & plague & mortal & menace \\
morale & meadow & gutter & grocer & sturdy & candle \\
\hline
\end{tabular}

Note-DN, deletion neighbor.

(Manuscript received March 16, 2004;

revision accepted for publication January 18, 2005.) 